\title{
Histochemical and Morphological Characteristics of the Hassall's Corpuscles. The Stress Affects Involution of the Ectopic Intra-Thyroidal and the Normal Position Thymus and Morphological Changes of the Hassall's Corpuscles
}

Satoshi Furukawa*, Lisa Wingenfeld, Satomu Morita, Akari Takaya, Tokiko Nakagawa, Ikuo Sakaguchi, Wakoto Matsuda and Katsuji Nishi

Department of Legal Medicine, Shiga University of Medical Science Setatukinowa-cho, Ohtsu city 520-2192, Shiga, Japan

\begin{abstract}
We encountered three cases of the intra-thyroidal ectopic thymus at autopsy and also performed three autopsy cases consisted of mothers and children, and children with severe stress. The morphological and immune histochemical characteristics of the Hassall's corpuscles were examined in those in ectopic and normal positioned thymus, and in the thymuses obtained from mothers and children, and children with severe stress. The Hassall's corpuscles obtained from ectopic and normal positioned thymus showed resemble morphology and stainability with antibodies. These results indicate the Hassall's corpuscles in the intra-thyroidal ectopic thymuses also possess the biological significance.
\end{abstract}

Keywords: Morphology of Hassall's corpuscle; Antigen expression in the Hassall's corpuscle; Ectopic thymus

\section{Introduction}

Embryologically, the thymus is derived from all 3 germinal layers and arises primarily from the third pharyngeal pouch [1]. It descends from the piriform sinus and passes through the thyrohyoid membrane and out along the carotid sheath bilaterally. The primordium fuse in the midline and the thymus then descends into its usual anatomical position in the anterior mediastinum, where there is regression of the endoderm and lymphocyte invasion to form the characteristic Hassall's corpuscles. The thymus occasionally fails to descend into the mediastinum. Usually, the main body of the thymus descends, leaving a strip of thymus tissue or a fibrous remnant along the line of embryonic descent [2].

We encountered three cases of the intra-thyroidal ectopic thymus at autopsy in the last four years (Figure 1).The reactivity of the Hassall's corpuscles with antigens used was summarized in other else [3] in which we described that there were at least four different morphology of the Hassall's corpuscles, that is, small solid type, solid type, mosaic type, and cystic type.

\section{Case Reports}

The thymus tissues were obtained the ectopic and normal positioned thymus from 5 months-girl who died due to infectious bronchial pneumonia and 3 months boy who died due to infectious bronchial pneumonia and 16 years-old girl who was found death in the scene of a fire of her house with a 43 years old her mother and a 13 years old younger sister. The thymus tissues additionally obtained from two pairs of mother and girl or boy was also used in this study. One pair consisted of a 37 years old pregnant woman, who died due to bleeding from the uterus and a newly born girl who died due to chest compression by mother's arm. Other case consisted of a 38 years old mother who died by hanging and a 10 years old boy who died due to strangulation by his mother using a rope. Histological examination was also performed in boy and girl who died due to hunger or sepsis by burn in an abusing, and a 14 year old boy who died due to subdural hemorrhage after two weeks of his head injury. The tissue sections were fixed in formalin and embedded in paraffin. Two sections from each sample of ectopic and normal position thymus and one from usual thymuses were stained using the haematoxylin-eosin (HE), to observe the overall structural aspects, number, shape and particulars of the Hassall's corpuscles. Additional sections from each sample were immunohistochemically stained. The data concerning the antibodies used in this study was shown in table 1 . Staining procedure was performed by using Histofin SAB-PO kit (Nichirei Japan). Assessment of the staining results was carried out with the Nikon microscope system and pictures were edited in JPEG format.

\section{Results}

Morphology of the Hassall's corpuscle in the ectopic and normal positioned thymuses

The ectopic thymuses composed of cortical and medulla portions,
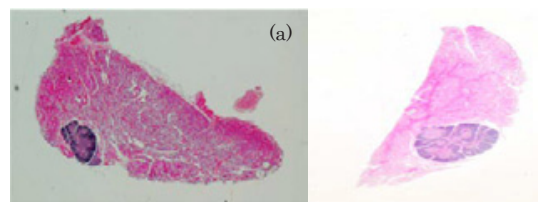

(b)

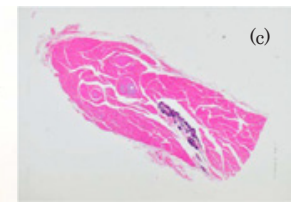

Figure 1: Three cases of the intra-thyroidal ectopic thymuses. HE staining showed that the thyroidal tissue covered a small nodule with blue color, where the thymus tissues were contained. (a); from 5 months girl, (b); from 3 months boy, and (c); from 16 years old girl.

*Corresponding author: Dr. Satoshi Furukawa, MD, Department of Legal Medicine, Shiga University of Medical Science, Setatsukinowa, Otsu City, Shiga 520-2192, Japan, Tel: +81-77-548-2200; E-mail: 31041220@belle.shiga-med.ac.jp

Received August 07, 2012; Accepted September 25, 2012; Published September 27, 2012

Citation: Furukawa S, Wingenfeld L, Morita S, Takaya A, Nakagawa T, et al. (2012) Histochemical and Morphological Characteristics of the Hassall's Corpuscles. The Stress Affects Involution of the Ectopic Intra-Thyroidal and the Normal Position Thymus and Morphological Changes of the Hassall's Corpuscles. J Forensic Res 3:165. doi:10.4172/2157-7145.1000165

Copyright: (c) 2012 Furukawa S, et al. This is an open-access article distributed under the terms of the Creative Commons Attribution License, which permits unrestricted use, distribution, and reproduction in any medium, provided the original author and source are credited. 
Citation: Furukawa S, Wingenfeld L, Morita S, Takaya A, Nakagawa T, et al. (2012) Histochemical and Morphological Characteristics of the Hassall's Corpuscles. The Stress Affects Involution of the Ectopic Intra-Thyroidal and the Normal Position Thymus and Morphological Changes of the Hassall's Corpuscles. J Forensic Res 3:165. doi:10.4172/2157-7145.1000165

Page 2 of 4

\begin{tabular}{|c|c|c|c|c|c|c|}
\hline Antibody & Maker & Clone & Species & AG-retrieval & incubation & AB-dilution \\
\hline CB & EPITOMICS & EPR4323: & $\mathrm{R}$ & autoclave & overnight & $1: 400$ \\
\hline$C D$ & santa cruz & E-7 & $M$ & autoclave & overnight & $1: 200$ \\
\hline eNOS & Gene Tex & polyclonal: & $\mathrm{R}$ & autoclave & overnight & prediluted \\
\hline GC-R & Novus & BuGR2: & $M$ & autoclave & overnight & $1: 400$ \\
\hline MUC 4 & abcam & $8 G-7$ & $M$ & autoclave & overnight & $1: 400$ \\
\hline SIRT1 & Novus & E104: & $\mathrm{R}$ & autoclave & overnight & $1: 400$ \\
\hline HSP70 & santa cruz & polyclonal: & G & autoclave & overnight & $1: 400$ \\
\hline P53 & santa cruz & FL-393: & G & autoclave & overnight & $1: 400$ \\
\hline $\mathrm{HCK}$ & Nichirei & 34ßE12: & $M$ & retrievalsolution & overnight & prediluted \\
\hline VEGF & Milipore & $\mathrm{JH} 121$ & M & autoclave & overnight & $1: 400$ \\
\hline CD15 & Dako & C3D-1: & $M$ & non & overnight & $1: 200$ \\
\hline Ley & Dako & BM-1: & M & non & overnight & $1: 200$ \\
\hline Lea & Calbiochem & T174 & M & non & overnight & $1: 200$ \\
\hline Leb & Calbiochem & T218 & $M$ & non & overnight & $1: 200$ \\
\hline A & Ortho & & $M$ & non & overnight & $1: 400$ \\
\hline B & Ortho & & $M$ & non & overnight & $1: 400$ \\
\hline $\mathrm{H}$ & MONOSAN & A-70/A9 & $M$ & non & overnight & $1: 100$ \\
\hline
\end{tabular}

Table 1: Characteristics of the antibodies used.

and Hassall's corpuscles were recognized in all the cases of ectopic thymus, and their number, size and morphological aspect were differ depend on each individuals, however morphological aspects were similar between ectopic and normal positioned thymuses within individuals. The Hassall's corpuscles observed in the thymus from 16 years old girl mainly showed small type one and those from girl and boy mainly showed the mosaic type with moderate size, although they were completely younger. This result indicates that the morphology of the Hassall's corpuscle was unrelated to the age of the individuals.

The Hassall's corpuscle in both thymuses obtained from each individuals showed similar reactivity with antibodies used in this study (Figure 2), showing the Hassall's corpuscle in the ectopic thymus possessed similar activity to those in normal positioned thymuses.

\section{Thymic involution}

The thymus involution was intensively increasing with age in a family consisted of mother and two daughters as shown in figure 3. No thymic tissue was detected in a boy with hunger (Figure 4) and intensive involution of the thymus was observed in an abused girl and a boy with head injury. In this boy, no thymus was detected at autopsy and microscopic level. The results indicate that thymic involution normally occur with ageing, however stress can intensive progress the thymic involution irrespectively with age.

\section{Morphology of the Hassall's corpuscles in mothers and children, and in individuals with severe stress}

The Hassall's corpuscles observed in thymus from mother and two daughters showed small with immature and no calcification, no mosaic pattern and no large one. These phenomena were detected in other mothers and child groups as shown in figure 5 . When the Hassall's corpuscles from a mother showed the relatively large sized one or mosaic one, those from child also showed large one or mosaic morphology. Number of Hassall's corpuscles with calcification was increased in individuals with severe stress and/or being abused, as shown in figure 6 . The thymus obtained from 4 years old girl who had been receiving sever abuse from her mother and father for over one year, and died due to sepsis caused by burn, showed intensive involution of the thymus and calcification of the Hassall's corpuscle, on the other hand the thymus from 14 years old boy showed slight involution of the thymus and moderate calcification of the Hassall's corpuscles, showing that the calcification may occur prior to the involution described above. The Hassall's corpuscles with calcification showed no reactivity with antibodies including anti-ABO blood antigens in the calcification part.

\section{Discussion}

The thymus appears during the sixth week of gestation and is primarily derived from the ventral wing of the third pharyngeal pouch with possible minor rudimentary portions derived from the fourth pharyngeal pouch [1]. The intra-thyroidal ectopic thymes is uncommon and can be misdiagnosed as a thyroid lesion resulting in unnecessary further evaluation or treatment in the clinic [4]. Ito et al. [5] described that in some cases intra-thyroidal thymoma was thought to originate from ectopic thymic tissue or embryonic thymic rest in or adjacent to the thyroid. Concerning the incidence of the ectopic intrathyroidal thymus there are several reports showing different frequency, that is, from $0.03 \%$ to $3.14 \%[6,7]$.

Steinman et al. [8] showed that the lymphoid component within the true Thymic Epithelial Space (TES) begins to involute shortly after birth and decreases $\sim 3 \%$ /yr through middle age (35-45 years of age) and continues to decrease $\sim 1 \% / \mathrm{yr}$ throughout the rest of life. The expansion of the non-thymopoietic Perivascular Space (PVS) such as adipocytes, peripheral lymphocytes and stroma, in the thymus with age results in a shift in the ratio of true TES to PVS, with the TES shrinking to $<10 \%$ of the total thymic tissue by 70 years of age. George and Ritter [9] described that the thymus would completely cease to produce new T cells at $\sim 105$ years of age.

The Hassall's corpuscles are unique, functionally active, multicellar components of the non-lymphocytic microenvironment of the thymus and participate in its physiological activities [10]. Since first describing of Hassall's corpuscle by Arthur Hill Hassall in 1849 [11], the Hassall's corpuscles were suggested to represent the 'graveyard' for dead thymocytes or the 'privileged area' for the maturation of medullary thymocytes [12].

However other studies suggested that the Hassall's corpuscle are active in many antigen expression, cell signaling, transcription, and metabolism mediated by cytokines or growth factor receptors [1315]. Watanabe et al. [16] revealed that Hassall's corpuscles have a critical role in debdritic-cell-mediated secondary positive selection of 
Citation: Furukawa S, Wingenfeld L, Morita S, Takaya A, Nakagawa T, et al. (2012) Histochemical and Morphological Characteristics of the Hassall's Corpuscles. The Stress Affects Involution of the Ectopic Intra-Thyroidal and the Normal Position Thymus and Morphological Changes of the Hassall's Corpuscles. J Forensic Res 3:165. doi:10.4172/2157-7145.1000165

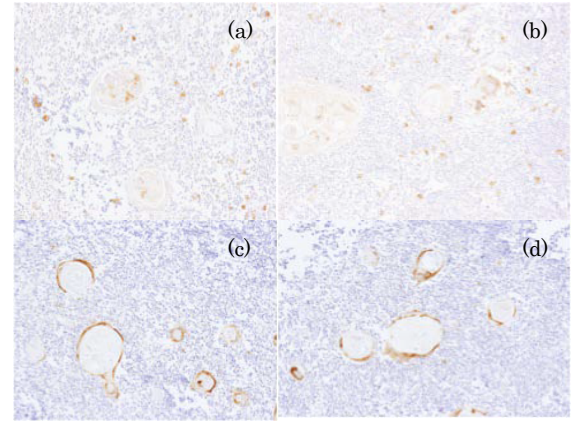

Figure 2: The reactivity of the Hassall's corpuscles in normal positioned and ectopic thymus with antibodies. The Hassall's corpuscles showed the same reactivity. Anti glucocoticoid receptor antibody stained the Hassall's corpuscles from normal positioned thymus (a) and (b) from 5 months girl. Anti MUC 4 also stained the Hassall's corpuscles from normal positioned thymus (c) and (d) from 3 months boy.

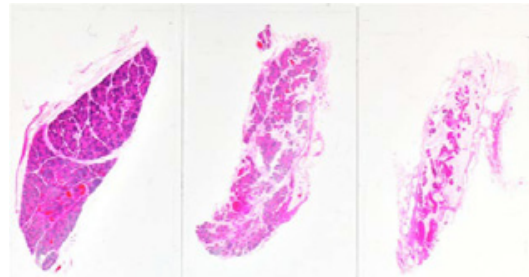

Figure 3: The thymus stained by HE, were obtained from a mother and her daughters. The thymus (a) from the mother (42 years old) shows progressive involution, the thymus from elder daughter (16 years old) shows slight involution and the thymus from younger daughter (14 years old) shows parenchyma tissue.

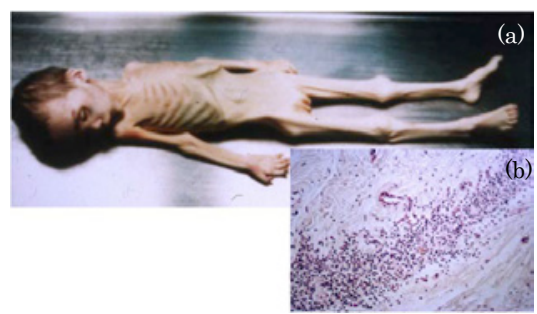

Figure 4: Complete involution of the thymus observed in a boy. A boy died due to hunger (a), and the complete involution of the thymus that remained small amount of lymphocytes (b), was observed.

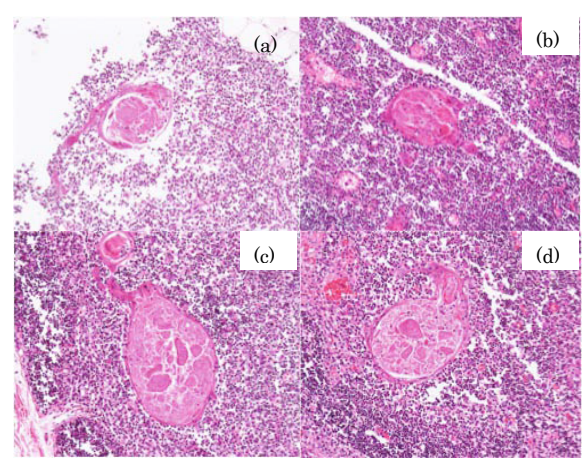

Figure 5: The morphology of the Hassall's corpuscle in couples of mother/boy $(a, b)$ and mother/daughter (c,d). The Hassall's corpuscle in these individuals showed mosaic pattern, those from new born infant (d) also showed mosaic one.

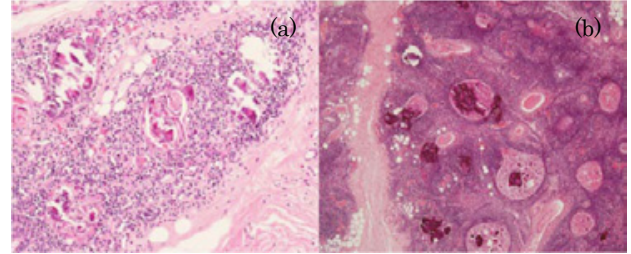

Figure 6: The Hassall's corpuscles with calcification and thymic involution. (a); Many number of Hassall's corpuscles from 4 years old girl who died due to sepsis, are changed into calcification, and involution of the thymus was sever. (b); Hassall's corpuscles with partial calcification were observed in a 14 years old boy who died due to subdural bleeding after two weeks of his head injury. The involution was not severe.

medium-to-high affinity self-reactive $\mathrm{T}$ cells, leading to the generation of CD4+ CD25+ regulatory $\mathrm{T}$ cells within the thymus. In this study we examined the morphological difference and the localization of several antigens in the Hassall's corpuscles both in intra-thyroidal and normal positioned of thymus.

The results indicate that the Hassall's corpuscles in the ectopic thymus also keep their role as the secretory parts for Thymic Stromal Lymphopoietin (TSLP). Fukunaga et al. [17] reported that the involution of the thymus can become an indication for child abuse and neglect, the ratio of the Hassall's corpuscles with calcification may also indicate the degree and time of stress that inflicted the healthy situation of children. Further studies are necessary to solve this subject.

\section{Acknowledgement}

The Ethics Committee of the Shiga University of Medical Science approved this study. We thank Shiga University of Medical Science for supporting this project and giving permission to publish the article.

\section{References}

1. Tovi F, Mares AJ (1978) The aberrant cervical thymus: Embryology, pathology, and clinical implication. Am J Surgery 136: 631-637.

2. Harman NB (1901) "Socia thymi cervicalis," and thymus accessorius. J Anat Physiol 36: 47-53.

3. Takaya A, Furukawa S, Wingenfeld L, Sakaguchi I, Nakagawa T, et al. (2012) Immunohistochemical characteristics of the Hassall's corpuscles. Jpn J Legal Med 66: 94.

4. Gimm O, Krause U, Wessel H, Finke R, Dralle H (1997) Ectopic intrathyroida thymus diagnosed as a solid thyroid lesion: case report and review of the literature. J Pedatr Surg 32:1241-1243.

5. Ito Y, Miyauchi A, Nakamura Y, Miya A, Kobayashi K, et al. (2007) Clinicopathologic singnificance of intrathyroidal epithelial thymoma/carcinoma showing thymus-like differentiation: a collaborative study with member institutes of the Japanese Society of Thyroid Surgery. Am J Clin Pathol 127: 230-236.

6. Bale PM, Sotelo-Avila C (1993) Maldescent of the thymus: 34 necropsy and 10 surgical cases, including 7 thymuses medial to the mandible. Pediatr Pathol 13: $181-.190$.

7. Avula S, Daneman A, Navarro OM, Moineddin R, Urbach S, et al. (2010) Incidental thyroid abnormalities identified on neck US for non-thyroid disorders Pediatr Radiol 40:1774-1780.

8. Steinman GG, Klaus B, Mueller-Hermelink HK (1985) The involution of the ageing human thymic epithelium is independent of puberty. A morphometric study. Scand J Immunol 1985: 22: 563-575.

9. George AJ, Ritter MA (1996) Thymic involution with ageing: obsolescence or good housekeeping? Immunol Today 17: 267-272.

10. Bodey B, Bodey B, Siegel SE, Kaiser HE (2000) Novel insights into the function of the thymic Hassall's bodies. In Vivo 14: 407-418.

11. Hassall AH (1849) The microscopic anatomy of the human body in health and disease, Plate LXI, London. 
Citation: Furukawa S, Wingenfeld L, Morita S, Takaya A, Nakagawa T, et al. (2012) Histochemical and Morphological Characteristics of the Hassall's Corpuscles. The Stress Affects Involution of the Ectopic Intra-Thyroidal and the Normal Position Thymus and Morphological Changes of the Hassall's Corpuscles. J Forensic Res 3:165. doi:10.4172/2157-7145.1000165

12. Senelar R, Escola MJ, Escola R, Serrou B, Serre A (1976) Relationship between Hassall's corpuscles and thymocytes fate in guinea-pig foetus. Biomedicine 24: 112-122

13. Ushiyama I, Kouno A, Yamamoto Y, Nishimura A, Ito N, et al. (1997) Hassall's Ccorpuscle functuon as the secretory cell groups. Forensic Pathology 3: 111117.

14. Romagnani P, Annunziato F, Manetti R, Mavilia C, Lasagni L, et al. (1998) High CD30 ligand expression by epithelial cells and Hassal's corpuscles in the medulla of human thymus. Blood 91: 3323-3332.
15. Nishio H, Matsui K, Tsuji H, Tamura A, Suzuki K (2001) Immunolocalization of the mitogen-activated protein kinase signaling pathway in Hassall's corpuscles of the human thymus. Acta Histomech 103: 89-98.

16. Watanabe N, Wang YH, Lee HK, Ito T, Wang YH, et al. (2005) Hassall's corpuscles instruct dendritic cells to induce $\mathrm{CD} 4+\mathrm{CD} 25+$ regulatory $\mathrm{T}$ cells in human thymus. Nature 436: 1181-1185.

17. Fukunaga T, Mizoi Y, Yamashita A, Yamada M, Yamamoto Y, et al. (1992) Thymus of abused/neglected children. Forensic Sci Int 53: 69-79. 